\title{
Fibroblast growth factor 2: good or bad guy in the joint?
}

\author{
Tonia L Vincent* \\ See related research by Yan et al., http://arthritis-research.com/content/13/4/R130
}

\begin{abstract}
Fibroblast growth factor 2 (FGF2) is a highly abundant growth factor found within the pericellular matrix of articular chondrocytes, but studies investigating its role have been conflicting. The paper reported by Yan and colleagues in the previous issue of Arthritis Research \& Therapy proposes that differences in responses to FGF2 are most likely due to changes in the balance between the two major articular cartilage FGF receptors, FGFR1 and FGFR3. They show that the catabolic and anti-anabolic effects of FGF2 are mediated primarily through FGFR1 whereas the beneficial effects are through FGFR3. This balance is dynamic and is altered in disease and following growth factor stimulation in vitro.
\end{abstract}

Fibroblast growth factor (FGF)2 is a ubiquitously expressed pleiotropic growth factor with many recognised cellular targets and tissue effects. Its role in articular chondrocytes and mesenchymal cells has been extensively studied, but published data are plagued by apparent contradiction and inconsistency. The paper by Yan and colleagues reported in the previous issue of Arthritis Research \& Therapy proposes a novel and elegant explanation for these seemingly disparate and conflicting findings [1]. FGFs signal through one of four FGF receptors (FGFRs), and the authors show that the catabolic effects of FGF2 (induction of proteases such as matrix metalloproteinase 13 (MMP13) and ADAMTS5 (a disintegrin and metalloproteinase with thrombospondin motifs 5)), as well as suppression of matrix synthesis, are specifically mediated through FGFR1 ligation. They propose that FGF2 signalling via FGFR3, the other major cell surface receptor on chondrocytes, leads to a dominant anabolic

*Correspondence: tonia.vincent@kennedy.ox.ac.uk Kennedy Institute of Rheumatology, University of Oxford, 65 Aspenlea Road, London, W6 8LH, UK drive. This is supported by the finding that FGF18, which is unusual because it is less promiscuous than FGF2 and selectively activates only FGFR3 [2], is able to stimulate proteoglycan synthesis. Other reports have confirmed the anabolic nature of FGF18 and an ongoing clinical trial of intraarticular injection of recombinant FGF18 in osteoarthritis is currently being conducted by Merck Serono (reviewed in [3]).

Our own studies have demonstrated that FGF2 is stored within the pericellular matrix of chondrocytes attached to the heparan sulphate chains of perlecan [4]. This pool of growth factor is liberated upon injury or mechanical compression of the matrix [5]. We demonstrated that, at least in young mice, the overall effect of FGF2 in the joint is benign. Indeed, mice lacking FGF2 (FGF2 null mice) developed accelerated osteoarthritis (with age and following surgical joint destabilisation), and this could be reversed by delivery of subcutaneous recombinant FGF2 [6]. When gene expression analysis was performed in FGF2 null joints after induction of osteoarthritis, there was a super-induction of ADAMTS5 mRNA, suggesting that FGF2 normally suppresses ADAMTS5. This was consistent with the ability of FGF2 to suppress interleukin-1-driven aggrecanase activity in normal human articular cartilage explants [7]. These persuasive anti-catabolic activities of FGF2 could well be dependent upon differences in the balance of FGFRs in these tissues, although it is also worth noting that in vivo many other cells within the joint respond to FGF2 (besides chondrocytes) and these could be contributing to the increased disease seen in FGF2 null mice. All the same, these studies alert us to the possibility that receptor expression differs between different tissues, ages of tissue and possibly even species.

The authors also make the interesting observation that the FGFR balance is dynamic. FGF2 itself is able to suppress the expression of FGFR3, whilst bone morphogenetic protein (BMP7) is able to augment expression. This does raise the question as to whether receptor expression is significantly altered when cartilage is explanted and cells are isolated. As FGF2 itself is released upon explantation of cartilage [5], this process may affect 
the balance of FGFRs in the resulting assay material, perhaps leading to skewing of the natural balance of receptors and tending towards a more catabolic phenotype in cultured cells/explants in vitro. It is not known whether FGF18 and BMP7 are also endogenous pericellular matrix-bound proteins, and whether they too are liberated in response to injury. FGF18 has been shown to bind the core protein of perlecan in vitro [8] and it is able to form FGFR3-activating complexes with chondrocytederived perlecan [9]. BMP7 is made by chondrocytes and is known to bind to heparan sulphates and fibrillin, and thus may also be bound within the pericellular matrix [10]. The controlled release of these factors within the immediate vicinity of the cell surface could, like FGF2, represent a rapid and efficient way of controlling chondrocyte responses.

The question of whether the FGFR balance changes with age and with disease is an interesting and important one. Yan and colleagues show that there is a significant decrease in FGFR3 expression in human osteoarthritis cartilage compared to tissue from normal post mortem samples, which suggests that release of pericellular FGF2 in diseased cartilage may lead to predominantly catabolic effects and the benefits of exogenous FGF18 will no longer be realisable. The extent to which this will limit the utility of FGF18 treatment in human disease will be disclosed in due course when the Merck Serono trial has been completed. In the meanwhile, an important focus will be to develop strategies that augment FGFR3 levels within diseased tissues in order to potentiate the beneficial effects of all FGF family members.

\section{Abbreviations}

ADAMTS5, a disintegrin and metalloproteinase with thrombospondin motifs; BMP, bone morphogenetic protein; FGF, fibroblast growth factor; FGFR, FGF receptor.
Competing interests

The author declares that she has no competing interests.

Published: 30 September 2011

\section{References}

1. Yan D, Chen D, Cool SM, van Wijnen AJ, Mikecz K, Murphy G, Im HJ: Fibroblast growth factor receptor 1 is principally responsible for fibroblast growth factor2-induced catabolic activities in human articular chondrocytes. Arthritis Res Ther 2011, 13:R130.

2. Davidson D, Blanc A, Filion D, Wang H, Plut P, Pfeffer G, Buschmann MD, Henderson JE: Fibroblast growth factor (FGF) 18 signals through FGF receptor 3 to promote chondrogenesis. J Bio/ Chem 2005, 280:20509-20515.

3. Beenken A, Mohammadi M: The FGF family: biology, pathophysiology and therapy. Nat Rev Drug Discov 2009, 8:235-253.

4. Vincent TL, McLean CJ, Full LE, Peston D, Saklatvala J: FGF-2 is bound to perlecan in the pericellular matrix of articular cartilage, where it acts as a chondrocyte mechanotransducer. Osteoarthritis Cartilage 2007, 15:752-763.

5. Vincent $T$, Hermansson M, Bolton M, Wait R, Saklatvala J: Basic FGF mediates an immediate response of articular cartilage to mechanical injury. Proc Natl Acad Sci U SA 2002, 99:8259-8264.

6. Chia SL, Sawaji Y, Burleigh A, McLean C, Inglis J, Saklatvala J, Vincent T: Fibroblast growth factor 2 is an intrinsic chondroprotective agent that suppresses ADAMTS- 5 and delays cartilage degradation in murine osteoarthritis. Arthritis Rheum 2009, 60:2019-2027.

7. Sawaji Y, Hynes J, Vincent T, Saklatvala J: Fibroblast growth factor 2 inhibits induction of aggrecanase activity in human articular cartilage. Arthritis Rheum 2008, 58:3498-3509.

8. Smith SM, West LA, Hassell JR: The core protein of growth plate perlecan binds FGF-18 and alters its mitogenic effect on chondrocytes. Arch Biochem Biophys 2007, 468:244-251.

9. Chuang CY, Lord MS, Melrose J, Rees MD, Knox SM, Freeman C, lozzo RV, Whitelock JM: Heparan sulfate-dependent signaling of fibroblast growth factor 18 by chondrocyte-derived perlecan. Biochemistry 2010, 49:5524-5532.

10. Rider CC, Mulloy B: Bone morphogenetic protein and growth differentiation factor cytokine families and their protein antagonists. Biochem J 2010, 429:1-12.

doi:10.1186/ar3447

Cite this article as: Vincent TL: Fibroblast growth factor 2: good or bad guy in the joint? Arthritis Research \& Therapy 2011, 13:127. 